\title{
TESS Lens-Bezel Assembly Modal Testing
}

\author{
Brandon J. Dilworth, Ph.D. and Alexandra Karlicek \\ MIT Lincoln Laboratory, 244 Wood Street, Lexington, MA 02420
}

\section{Nomenclature}

$\begin{array}{ll}\text { AR } & \text { Anti-Reflective } \\ \text { CCD } & \text { Charge-Coupled Device } \\ \text { EDU } & \text { Engineering Development Unit } \\ \text { FEA } & \text { Finite Element Analysis } \\ \text { FEM } & \text { Finite Element Model } \\ \text { FRF } & \text { Frequency Response Function } \\ \text { HeNe } & \text { Helium-Neon } \\ \text { MDOF } & \text { Multiple Degree of Freedom } \\ \text { MIT } & \text { Massachusetts Institute of Technology } \\ \text { RTV } & \text { Room Temperature Vulcanization } \\ \text { TESS } & \text { Transiting Exoplanet Survey Satellite } \\ \alpha_{E} & \text { Coefficient of thermal expansion of elastomer } \\ \alpha_{G} & \text { Coefficient of thermal expansion of glass } \\ \alpha_{M} & \text { Coefficient of thermal expansion of metal } \\ D_{G} & \text { Diameter of glass } \\ t_{E} & \text { Thickness of elastomer }\end{array}$

Abstract

The Transiting Exoplanet Survey Satellite (TESS) program, led by the Kavli Institute for Astrophysics and Space Research at the Massachusetts Institute of Technology (MIT) will be the first-ever spaceborne all-sky transit survey. MIT Lincoln Laboratory is responsible for the cameras, including the lens assemblies, detector assemblies, lens hoods, and camera mounts. TESS is scheduled to be launched in August of 2017 with the primary goal to detect small planets with bright host starts in the solar neighborhood, so that detailed characterizations of the planets and their atmospheres can be performed.

The TESS payload consists of four identical cameras and a data handling unit. Each camera consists of a lens assembly with seven optical elements and a detector assembly with four charge-coupled devices (CCDs) including their associated electronics. The optical prescription requires that several of the lenses are in close proximity to a neighboring element. A finite element model (FEM) was developed to estimate the relative deflections between each lens-bezel assembly under launch loads to predict that there are adequate clearances preventing the lenses from making contact. Modal tests using non-contact response measurements were conducted to experimentally estimate the modal parameters of the lens-bezel assembly, and used to validate the initial FEM assumptions.

\section{Key Words}

Non-contact measurements, modal analysis, model validation 


\section{Statement}

This work is sponsored by the National Aeronautics and Space Administration under Air Force Contract \#FA8721-05-C-0002. Opinions, interpretations, conclusions and recommendations are those of the author and are not necessarily endorsed by the United States Government.

\section{Background and Motivation}

The TESS instrument is a 7-element optical system with several of the lenses in close axial proximity to each other. The optical system is configured to function in the visible spectrum. A cross-section view of the 7-element system is shown in Fig. 1.

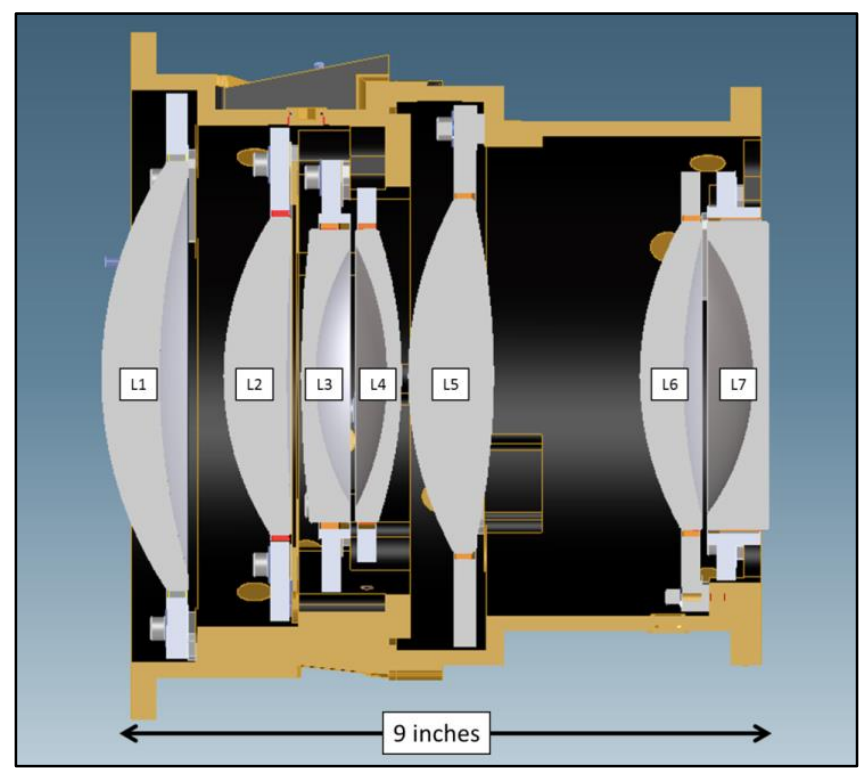

Fig. 1 Cross-section View of TESS Lens Barrel Assembly

The lenses are bonded to their associated aluminum bezels using a low-outgassing RTV (room temperature vulcanization) Silicone. The thickness of the RTV bonds is tailored to enable an athermal optical prescription. A common method to calculate necessary bond thicknesses to achieve this behavior follows Equation 1 [1].

$$
t_{E}=\frac{D_{G}\left(\alpha_{M}-\alpha_{G}\right)}{2\left(\alpha_{E}-\alpha_{M}\right)}
$$

The thickness of the RTV bond and the cured RTV mechanical properties are the drivers to the effective stiffness of the lensbezel interface. It is typical for elastomer materials, such as RTV, to not have handbook values for properties such as modulus which makes it difficult to estimate the joint stiffness with hand calculations. As-built cured RTV properties will vary from unit-to-unit due to factors such as mix ratios, mixing methods, cure temperature, and bakeout schedules. Because of this variation, it was desired to obtain measured results to inform the model of the estimated stiffness of the RTV joint.

A key interest early in the program was to determine if the RTV bonds, effectively relatively compliant springs, would be adequate to prevent the lenses from impacting each other during a launch environment. Early finite element analysis (FEA) models were used to perform the initial calculations of the sway-space of each lens during a launch loads analysis; sway-space 
being the total range of expected axial displacement of each lens. A 5-sigma factor and a factor a safety of 1.2 were applied to the displacement results from the launch loads FEA. The target metric (i.e. positive margin) was to ensure that each lens would remain within its allotted sway-space. The program desired to validate their FEA model with experiments, but the lens assemblies do not lend themselves to 'classical' dynamic measurement methods (i.e. one would not mount accelerometers to an optical element's surface). Multiple non-contact measurement methods were explored to accomplish model validation.

It should be noted that a complimentary need for the program was to not just validate the FEA model, but to also develop a test that would enable an in-situ estimate of the effective bond area relative to its nominal. If model validation was the only desire of the program, then one could imagine using mass mock-ups to analyze the dynamic stiffness of each bond thickness which could easily be accomplished with traditional measurement methods. However, this complimentary desire of the program led to explore other measurement options that could be executed with the actual optical elements as part of the test.

\section{Technical Approach}

As a baseline approach, a measurement method that could provide insight to the modal parameters of the first three modes of the lens-bezel assembly was desired. For all of the lens-bezel assemblies, it was expected that the first mode would be a piston mode (the lens moving planar in the axial direction) and the second and third modes would be tip and tilt. As the lens-bezel assemblies are nominally a symmetric geometry, the tip and tilt modes were expected to be very closely spaced modes. Analytically, the tip and tilt modes are double modes, but the expectation was that minor asymmetries as a result of fabrication tolerances would prevent these from being true double modes in the measurements. As with all systems with expected closely spaced modes, the experimental setup to include multiple reference locations in order to distinguish these modes [2].

As noted earlier, the desire to measure lens-bezel assemblies in-situ drove the need to explore non-contact measurement techniques. The first non-contact dynamic measurement method explored was laser Doppler vibrometry. Laser Doppler vibrometers measure Doppler shift using an interferometric technique which provides a direct measurand of relative surface velocity between the laser head and the target surface of the device under test [3]. A draw-back to laser vibrometry is the need to have a diffuse reflective surface at the target measurement locations. This is a difficult metric for lenses coated in the visible spectrum which include anti-reflective (AR) coatings (i.e. the HeNe red visible laser passes straight through the lens with a minimal return) without some kind of surface preparation. However, even with the need of a surface treatment, a significant advantage to the results of the laser vibrometer is to be able to scale the mode shape vectors relative to the physical structure during parameter estimation.

To demonstrate the feasibility of using a laser vibrometer for this type of test article, a modal impact test was conducted. An EDU lens-bezel assembly with representative RTV bonds was selected for testing. The lens-bezel assembly was mounted in a relatively massive fixture and the fixture was mounted to soft foam to estimate free-free boundary conditions. Two impact locations were selected, located at mounting bolt interfaces of the bezel (i.e. at the load path of the interface of the bezel), providing suitable results for reliably extracting the [expected] closely spaced tip-tilt modes. To prepare the optic such that it had a diffuse reflective surface, a developer was used which resulted in a flat white surface. The developer was also chosen as it was believed (and later proven) to be able to be cleaned from the optic. A photograph of the initial test setup is shown in Fig. 2 . 


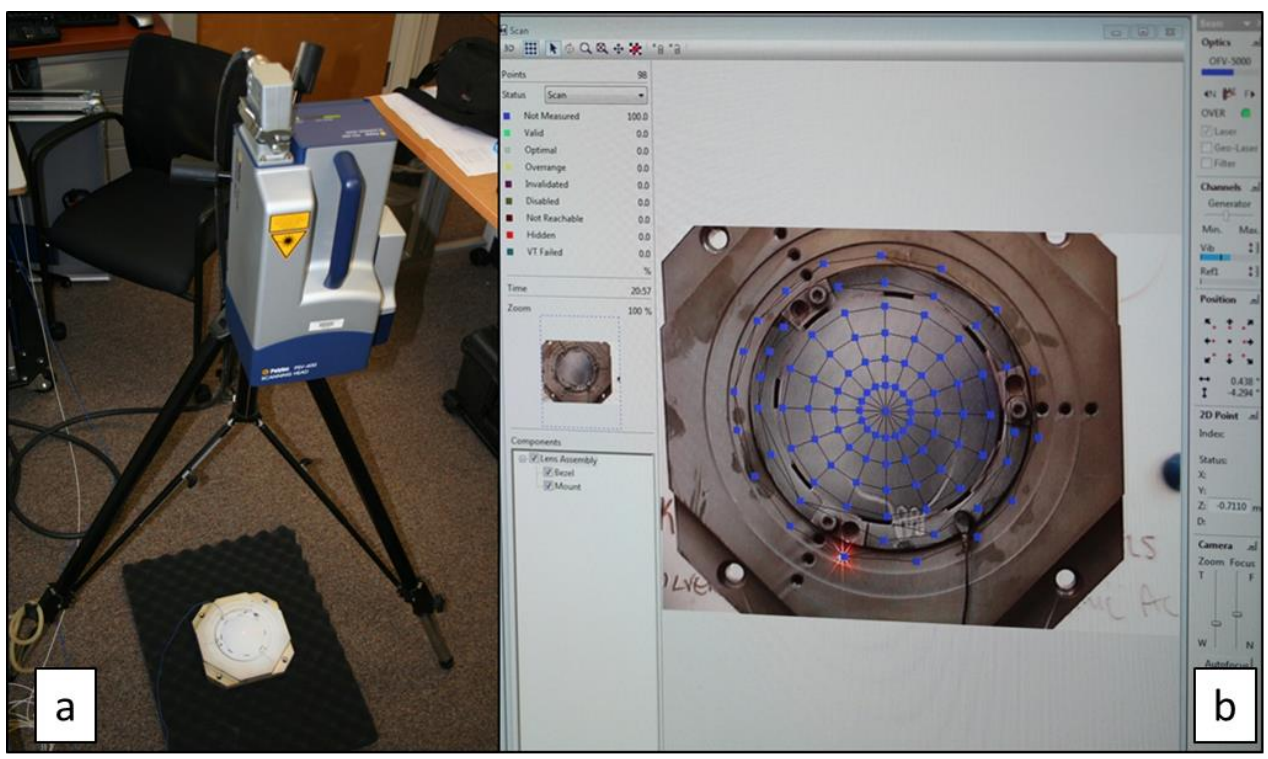

Fig. 2 Initial Laser Vibrometer Setup (a) and Initial Measurement Grid (b)

The laser vibrometer response measurement geometry was defined across the optic and around the bezel to provide confidence that the lens modes were separable from the fixture modes. For the initial measurements, a single axis accelerometer was also mounted on the bezel at the impact/input locations to validate driving point frequency response functions (FRF's). Detailed results will be presented in the following section, but the conclusion of the laser vibrometer testing was that the lens modes were readily separable from the fixture modes.

With the successful results of the laser vibrometer measurements, the program desired to develop a measurement technique where modifying the surface of the optic was not required. A couple of key considerations were made to evaluate a different approach. First, a non-contact method that did not require modifying the surface of the optic and still allowed modal parameter estimation was the primary interest. Second, scaled mode shapes were not considered necessary for evaluating the as-built condition of all the lens assemblies. Since the surface of the glass was considered to be very rigid and the glass would move as a rigid body relative to the bezel, a measurement method using microphones was considered. Using the microphones in the near-field, it was presumed that the pressure wave due to the motion of the glass would provide sufficient signal-to-noise to estimate the modal parameters of the lens-bezel assemblies. A subset of the response measurement locations used during laser vibrometer testing was selected for microphone tests. A photo of the initial measurement with a single microphone is shown in Fig. 3. Detailed results are discussed in the next section. 


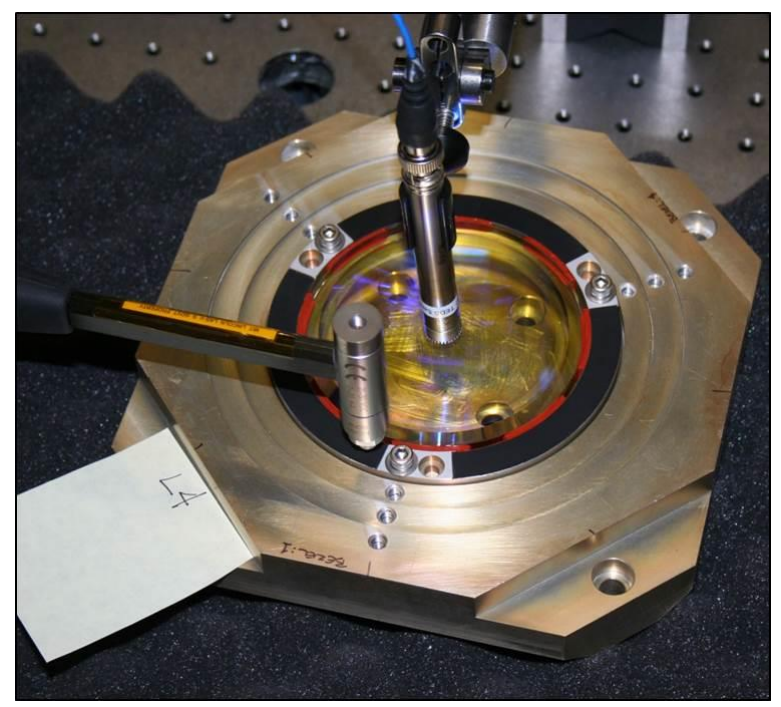

Fig. 3 Initial Microphone Setup

\section{Results}

Initial calculations indicated that the first three modes of the lens-bezel assemblies were expected to be in the frequency range of 150-600 Hz. To ensure that this entire frequency range was excited by the modal hammer, a Teflon tip was selected which resulted in an excitation bandwidth of $\sim 1600 \mathrm{~Hz}$. Softer tips were found not to excite the structure at a high enough frequency. Sampling parameters of the acquisition system were selected to match the excitation bandwidth, which is standard practice for any modal tests. Rectangular windowing was selected and a relatively unconventional excitation approached was used with a 2.5 second measurement window. Rather than a single impact, several impacts in a non-repeatable pattern were used to excite the structure. This method allowed an improved signal-to-noise ratio to be achieved in the measurement compared to a single impact. Initial measurements which justified this approach are shown in Fig. 4. Note that the FRF's have about half of the variance with the multiple impact method compared to a single impact for a given measurement average.

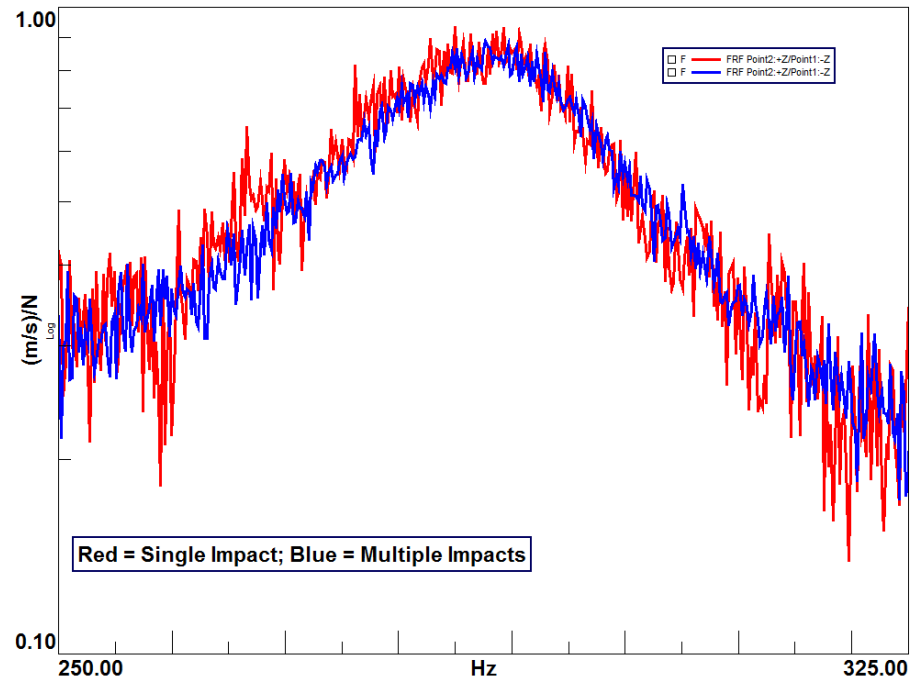

Fig. 4 FRF Comparison of Single Impact versus Multiple Impacts 
For the laser vibrometer measurements, an engineering unit (i.e. non-Flight) L4 lens-bezel assembly was used. Because it was a non-Flight lens, the program was willing to consider the use of a developer on the surface of the lens to provide a diffuse reflective surface for the vibrometer. It took several iterations to obtain a reliable return on the lens. Several coats of the developer were applied, and a beam dump (i.e. flat black paper) was placed behind the lens to limit the return of the laser light from the fixture. It was discovered that the back reflection from the fixture behind the lens interfered with the reflection of the front surface of the lens; the front surface of the lens being the measurand of interest. The beam dump measurably improved the signal-to-noise on the return beam.

Representative FRF's from the laser vibrometer measurements are shown in Fig. 5. These FRF's were used with a Time MDOF (multi-degree of freedom) curve fitter to estimate the modal parameters. The piston mode was readily observed as shown in Fig. 6(a), and the tip/tilt modes were also readily extracted as shown in Fig. 6(b). The curve fitted results were used to inform and update the stiffness calculation of the RTV in the lens-bezel assembly.

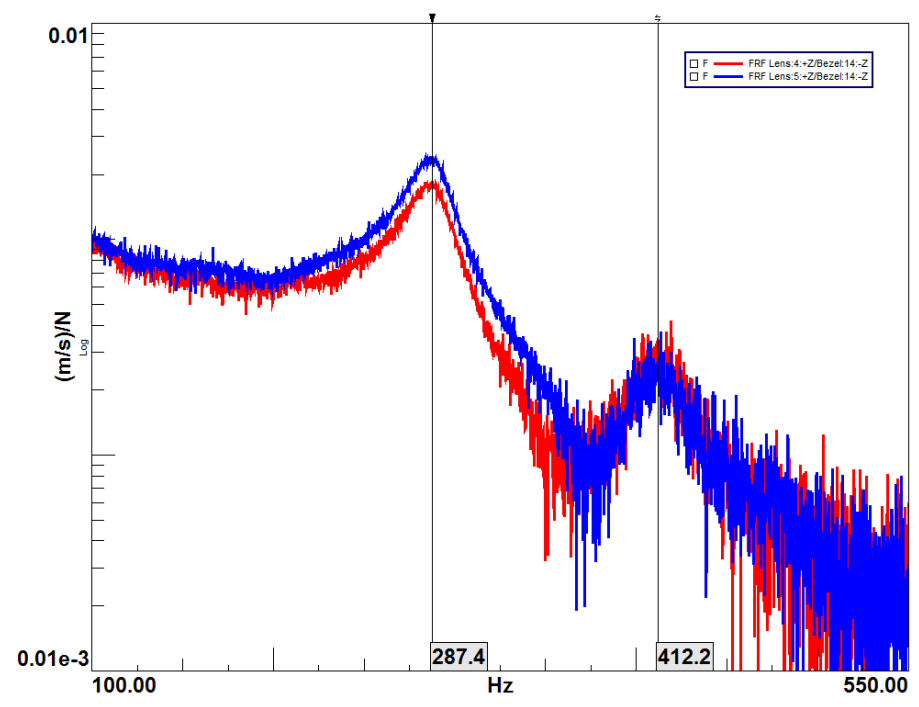

Fig. 5 Representative FRF's from Vibrometer Measurements

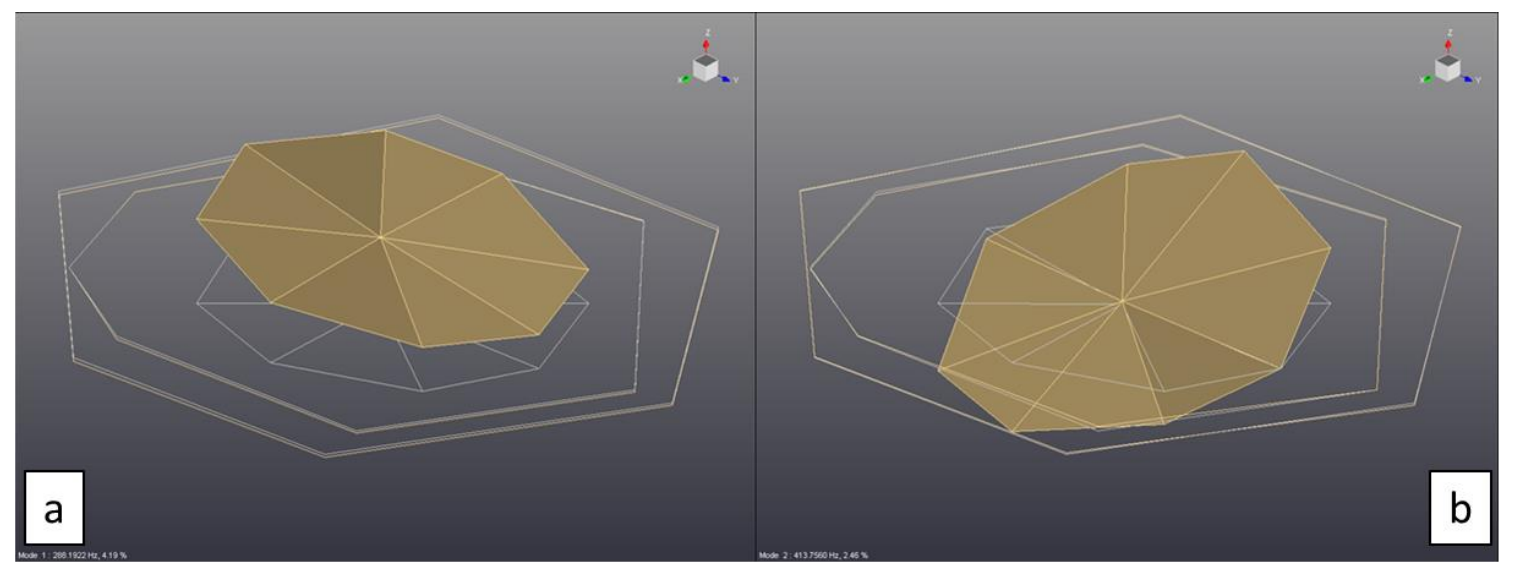

Fig. 6 Piston Mode (a) Tip/Tilt Mode (b) 
Testing of the engineering unit L4 lens-bezel assembly proved insightful enough that the program desired to perform insitu testing of all of the lenses (L1, L2, L3, L4, L5, L6, and L7). For these Flight-qualified lens-bezel assemblies, the microphone measurement technique was used. The same excitation method and sampling parameters used for the laser vibrometer tests was used for the microphone tests. Representative FRF's from the microphone approach are shown in Fig. 7. Although the FRF's are notably noisier than the results from the vibrometer measurements, the curve fitting was still able to successfully extract the modes of interest as shown in Fig. 8 (a) and Fig. 8 (b). These results compared very favorable to the updated calculations predicted by analysis.

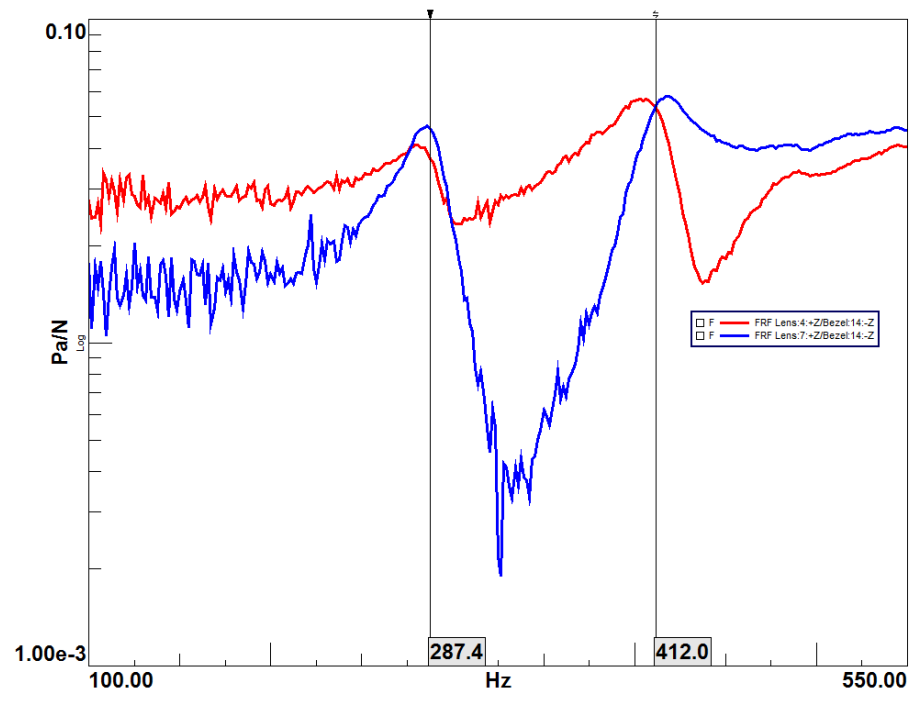

Fig. 7 Representative FRF's from Microphone Measurements

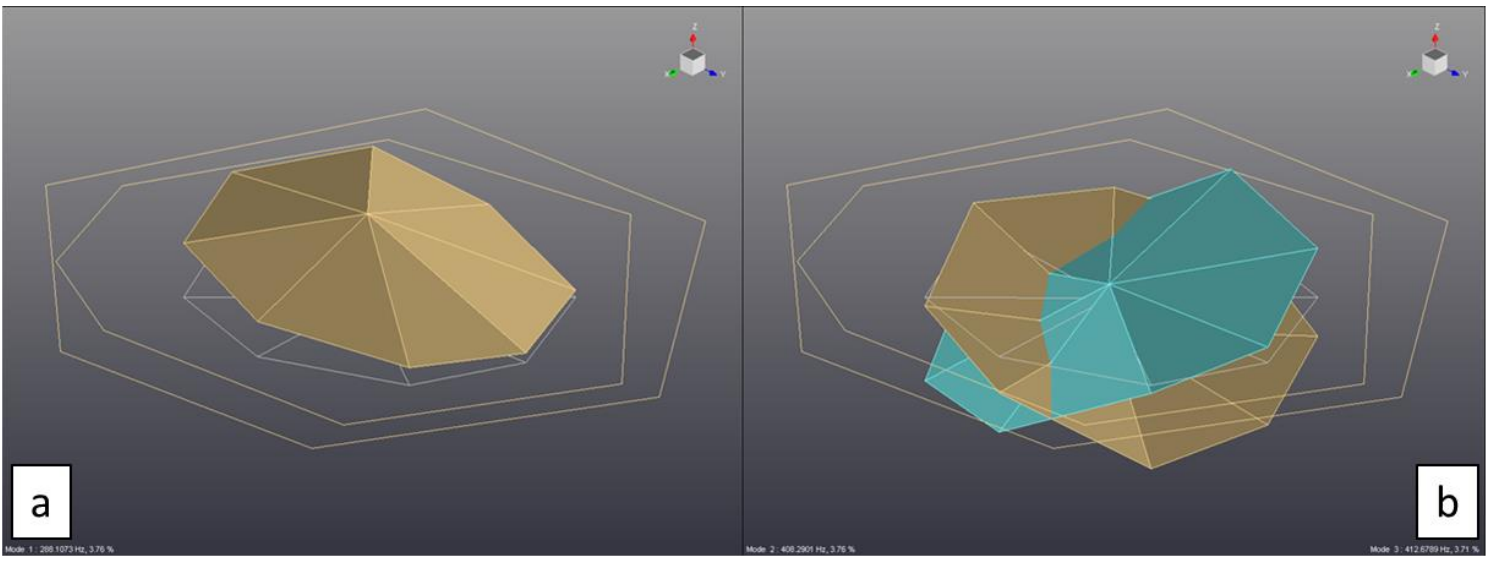

Fig. 8 Piston Mode (a); Tip/Tilt Mode (b)

These results showed that a reliable measurement method was demonstrated to perform an insitu estimate of the first three modes by using microphones. The microphones successfully accomplished this task without the need of modifying the surface of the lens in any way. The insight gained with this measurement prompted the program to desire to execute this measurement for all of the Flight lens-bezel assemblies as a means to verify the RTV bond. To prepare for testing five sets (4 sets of Flight and 1 set of spare) of lens assemblies (28 lens-bezel assemblies in total), multiple microphones and associated fixtures were used to shorten the test duration for each lens. A photo of the test setup for the Flight lens-bezel assemblies is shown in Fig. 9. 


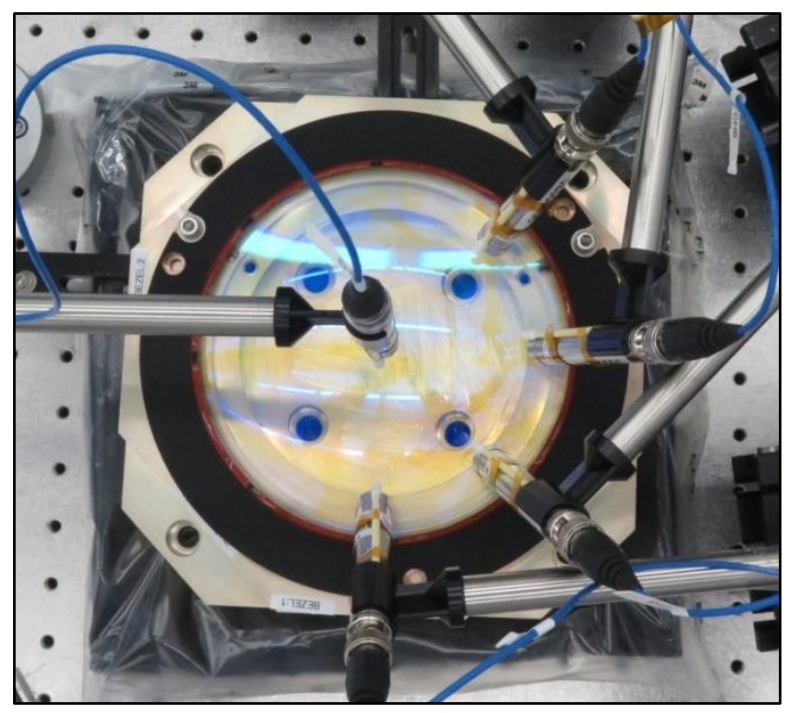

Fig. 9 Flight Lens Testing Setup

Testing of the Flight lens-bezel assemblies identified an unanticipated result. The first three modes were higher in frequency than predicted for RTV pads with $100 \%$ coverage. It was noted after some investigation that the Flight lens-bezel assemblies had been exposed to a vacuum bake at elevated temperatures where the non-Flight lens-bezel assemblies had not. With independent measurements of baked out RTV samples, it was found that the RTV stiffness did increase; validating the results of the modal testing. The results of the Flight lens-bezel assemblies were fed back into the FEA of the barrel assemblies and was determined that the sway space of each lens was reduced, as one would expect for modes at higher frequencies for a given load input.

\section{Conclusion}

A non-contact measurement method using microphones was developed to successfully determine the first three modes of the lens-bezel assemblies within the TESS barrel assembly. The microphone measurement method was proven on engineering unit assemblies and was readily transferrable to Flight unit assemblies. The developed measurement has been used on all Flight lens-bezel to prove the quality of the RTV bonds prior to final assembly.

\section{Bibliography}

1. Yoder, Paul, Jr., Opto-Mechanical Systems Design, Marcel Dekker, Inc, Optical Engineering Volume 9, pg 104, 1986.

2. Heylen, W., Lammens, S., Sas, P., Modal Analysis Theory and Testing, Katholieke Universiteit Leuven, pg B.2.9, 1997.

3. Polytec, "Theory Manual”, As of Software 8.8, 2011. 